\title{
CORRECTIONS TO THE FERMILAB RECYCLER FOCUSING WITH END SHIM CHANGES
}

\author{
D.E. Johnson, B.C. Brown, G.W. Foster, C. Gattuso, G.P. Jackson, C.S. Mishra, S.M. Pruss, \\ J.T. Volk, M-J Yang, FNAL*, Batavia, IL.
}

\begin{abstract}
The Recycler Ring Gradient Magnets were designed with end shims which correct the field shape (harmonic components up to 10-pole). A standard correction was employed at one end to correct the average properties. A variable correction was used at the other end to correct individual magnet variations during the fabrication process. Initial Recycler operation revealed that the quadrupole fields did not produce the desired tune. In part, this was due to the sextupole of the end-shim interacting with the curved orbit through the straight magnet. The standard end-shim was replaced on all regular cell gradient magnets. Details of these end corrections and the resulting successful modification of the lattice are described.
\end{abstract}

\section{INTRODUCTION}

The Recycler Antiproton Storage Ring[1] is comprised of 344 permanent magnet gradient dipoles and 94 permanent magnet quadrupoles. It is a FODO lattice with dispersion free straight sections. Originally, it included a 200-m high beta straight for electron cooling. A phase trombone was installed in the RR-60 long straight section, which contained 5 families of electromagnetic quads, to locally adjust the phase within the straight without perturbing the lattice outside the insert. It was originally designed to have +/- 0.5 units of tune adjustment. The design tunes (not using measured magnet harmonics) for a relaxed phase trombone (no current in trim quads) was Qx $=24.425$ and $\mathrm{Qy}=24.415$. Using the measured integrated multipoles for the quadrupole and sextupole the tunes are 24.408 and 24.387. Two families of electromagnetic sextupoles and skew quads were installed to control chromaticity and coupling.

\begin{tabular}{|c|c|c|c|c|c|}
\hline \multicolumn{2}{|c|}{ Magnet } & RGF & RGD & SGF & SGD \\
\hline $\mathrm{L}$ & $\mathrm{M}$ & 4.4958 & 4.4958 & 3.0988 & 3.0988 \\
\hline $\mathrm{B}_{0}$ & $\mathrm{~T}$ & 0.1375 & 0.1375 & 0.1330 & 0.1330 \\
\hline $\mathrm{B}_{1}$ & $\mathrm{~T} / \mathrm{m}$ & 0.3355 & -0.3238 & .66816 & -.6823 \\
\hline $\mathrm{b}_{1}$ & $\mathrm{X} 10^{-4}$ & 619.7 & -598.1 & 1276. & -1303. \\
\hline $\mathrm{B}_{2}$ & $\mathrm{~T} / \mathrm{m}^{2}$ & 0.3707 & -0.6417 & 0.0 & 0.0 \\
\hline $\mathrm{b}_{2}$ & $\mathrm{X} 10^{-4}$ & 8.696 & -15.05 & 0.0 & 0.0 \\
\hline
\end{tabular}

Table 1: Design specifications for the integrated dipole, quad, and sextupole fields in the four types of gradient magnets. The $b_{n} \times 10^{-4}=B_{n} x^{n} / n ! B_{0} @ 1 ”$.

\footnotetext{
*Operated by Universities Research Association Inc.,
} under contract with the U.S. Department of Energy.
Dipole correctors were installed where needed for injection/extraction manipulations. Table 1 lists the design values for the dipole, quadrupole and sextupole strengths of the gradient magnets. Tracking studies and dynamic aperture analysis were used to specify the magnetic field quality for both the systematic and random strength variations (up to sextupole) and harmonic content (up to 18-pole) .[2].

\section{MAGNET PRODUCTION AND INSTALLATION}

The integrated strength, longitudinal field distribution, and its behavior with temperature is determined by the quantity and placement of permanent magnet material and flux compensator material.[3] The body field shape (harmonics) is controlled by the pole-tip shape and placement. The expected field defects for the quadrupole and sextupole are on the order of $10^{-4}$ and less for higher orders.[3]

End shims (small $\sim$-inch sections of pole-tip) are used at each end of the gradient magnet to satisfy field strength and uniformity specifications. During production, a procedure was developed to measure and adjust the integrated field harmonics using a rotating coil harmonic probe on the magnet axis.[4] Average field defects were reduced by installing a "standard end-shim" on one end of the magnet. A "custom end-shim" was then used to bring each magnet into specifications. Table 2 lists a summary of the integrated, body, and end harmonics for the RGF (arc focusing) and RGD (arc defocusing) series.

\begin{tabular}{|c|c|c|c|}
\hline Region & Harmonic & RGF & RGD \\
\hline \hline \multirow{2}{*}{ Avg } & Quad & 619.79 & -597.89 \\
\cline { 2 - 4 } & Sext & 8.60 & -14.90 \\
\hline \multirow{2}{*}{ Body } & Quad & 620.21 & -596.87 \\
\cline { 2 - 4 } & Sext & 3.98 & -18.35 \\
\hline \multirow{2}{*}{$\begin{array}{c}\text { Label End } \\
\text { (custom shim) }\end{array}$} & Quad & 0.077 & -0.47 \\
\hline Sext & 2.43 & 1.96 \\
\hline $\begin{array}{c}\text { Other End } \\
\text { (std shim) }\end{array}$ & Quad & 0.5 & -0.55 \\
\hline
\end{tabular}

Table 2: Average measured values of quadrupole and sextupole of 108 RGF and 108 RGD gradient magnets.

Since the magnetic measurements utilize a straight probe, a prescription for translating the harmonics to the curved closed orbit was developed. The transverse offset 
was calculated to minimize the feed-down of the higher harmonics into the dipole, quadrupole, and sextupole terms.[5] Using this prescription, the gradient magnets were installed with a transverse offset of $7.46 \mathrm{~mm}$ and $3.29 \mathrm{~mm}$ for the long (RGF) and short (RGD) gradient magnets to maximize the aperture inside the magnet and minimize the feed-down effects. An analytical treatment of a curved orbit through a straight gradient magnet and the resultant transverse placement has revised offsets. [6]

\section{BEAM MEASUREMENTS}

Beam commissioning of the completed ring started late Spring of 1999. Circulating beam was established by the end of May by adding current to quad circuits in the phase trombone. Initial tune measurements, with current in the trombone quads, indicated that the horizontal tune was near the quarter integer resonance (24.26) and the vertical was near the half (24.46) and the chromaticities were $C x=-21$ and $C y=+13$ units, both far from design.

The phase advance per cell was measured on the first turn into the Recycler. [7] Two correctors in each plane were excited and difference orbits were used to calculate the phase advance between BPM locations. These measurements were compared with the Recycler model including all known harmonics (minus end-shim feeddown terms) in the gradient magnets and quads. For this data, all powered quadrupoles were off to measure the phase advance produced purely by the permanent magnets. Figure 1 shows the horizontal phase advancing slower than the model by 0.2 units $[2 \pi]$ and Figure 2 shows the vertical advancing faster than the model by roughly 0.18 units for a single turn. These figures indicate that this discrepancy is uniformly distributed and not localized.

Accelerator simulations with the design lattice (including measured multipoles) indicated the machine had an excess quadrupole in the RGF magnets by $\sim 1 \%$. When the gradients in the model were reduced by $1 \%$ and

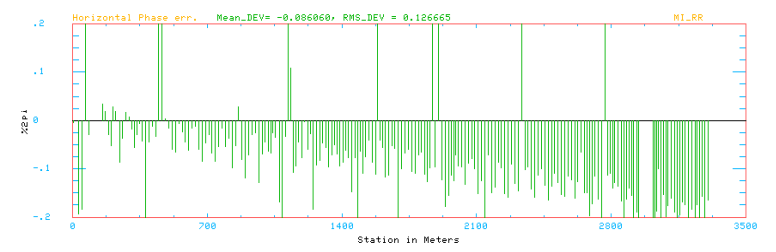

Figure 1: Initial Horizontal Phase advance (Note scale $+/-0.2$ )

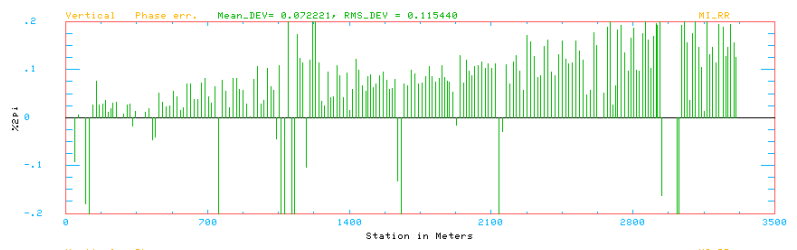

Figure 2: Initial Vertical phase advance (Note scale +/-0.2) the phase trombone quads were set to the currents used in the control system, the calculated tunes matched the measured tunes to within +/-.02 in both planes.

\section{RECYCLER PROBLEMS UNCOVERED}

A combination of accelerator measurements and simulations uncovered several problems in the Recycler as built. Each contributed to the measured tune and beta function errors, and chromaticity error in the machine.

- The harmonic content of the gradient magnet had been measured and reported as an integrated value. There were about 6 units (RGF) and 2.5 units (RGD) required sextupole in the end shims to satisfy the integrated sextupole specifications. The feed-down from this end-shim harmonics due to the sagatti offset was not taken into account. The magnitude and impact of this will be discussed below.

- An in situ beam pipe bake out system consisting of stainless steel strips on either side of the elliptical beam pipe was installed on all beampipe before it was inserted in the gradient magnets. The specifications did not include final magnetic properties, The selected material was 304 stainless sheets 20 mil thick and cut into 1 inch strips. After working, this material was magnetic. The effect of the material in the field of the gradient magnets was to perturb the body field harmonics. The magnitude of the harmonics was strongly dependent on the position of the beam pipe inside the gradient magnet.

- The incorporation of the high beta in the lattice implies large beta and strong quads. Tracking studies and dynamic aperture analysis showed a gradient tolerance of $\sim 0.1 \times 10^{-4}$. This insert contributed to phase advance and lattice error. T] he replacement or this insert is discussed elsewhere.[8]

\section{FEEDDOWN FROM END-SHIM}

The magnetic field expressed in terms of the harmonic components at a radius a is given by

$$
\mathrm{B}_{\mathrm{y}}(\mathrm{x})=\mathrm{B}_{0}\left(1+\mathrm{b}_{1}(\mathrm{x} / \mathrm{a})+\mathrm{b}_{2}(\mathrm{x} / \mathrm{a})^{2}+\ldots\right)
$$

where the $b_{1}$ and $b_{2}$ are the quadrupole and sextupole terms, $a$ is the radius at which the harmonic is measured (i.e. 1"), and $\mathrm{x}$ is the offset through the sextupole. The gradient may then be expressed as

$$
B^{\prime}=d B_{y}(x) / d x=B_{0}\left(b_{1} / a+2 b_{2} x / a^{2}\right) .
$$

From this, the contribution to the quadrupole is twice the total sextupole (contribution from shims on both ends) times the closed orbit offset at the shim location.

The impact of this quadrupole term may be estimated by summing up the additional gradient contribution (weighted by the local beta function at the shim location) 
at all 108 focusing (RGF) and all 108 defocusing (RGD) locations by

$$
\delta v=(1 / 4 \pi) \Sigma_{i} \beta_{i} B^{\prime}{ }_{i} /(\beta \rho)
$$

where $\beta_{i}$ is the beta function at the shim, $\mathrm{B}{ }_{\mathrm{i}}$ is the gradient due to sextupole feed-down, and $\beta \rho$ is the magnetic rigidity.

This summation was performed numerically in MAD and the results yielded that the horizontal tune is shifted down by 0.15 units and the vertical tune is shifted up by 0.12 units. This is, however still in the original design range of the phase trombone although we desire not to use the phase trombone to correct this feed-down.

We calculate the total end-shim sextupole contribution for each type of magnet (RGF and RGD) as the sum of the standard shim and the average of the custom shim. Table 2 shows the total $b_{2}$ at end shims for the RGF was 4.6 units and the RGD was 3.5 units. The feed-down to the quadrupole is then 2.7 and 2.1 units added to the original RGF and RGD quadrupole term, respectively.

We revise the shim to only correct the feed-down from the end-shim sextupole and we do not try to correct for other errors that might impact the base machine tune.

These gradient contributions were added to the "standard shim" of each type producing a new set of shims to be installed. Table 3 shows the harmonic content of the new shims installed in January 2000.

\begin{tabular}{|c|c|c|c|}
\hline Region & Harmonic & RGF & RGD \\
\hline \hline Other End & Quad & 3.2 & 1.55 \\
\hline & Sext & 2.8 & 1.49 \\
\hline
\end{tabular}

Table 3: New harmonic for the revised standard end shim

\section{POST RETROFIT BEAM MEASUREMENTS}

After removing the heater tape and replacing the standard shim, the tune and chromaticity were measured with the phase trombone quads and chromaticity sextupoles off. The bare machine circulating beam tunes are now measured to be $\mathrm{Qx}=24.435$ and $\mathrm{Qy}=24.351$. The model predictions for the tunes (which incorporate the feed-down) are $\mathrm{Qx}=24.408$ and $\mathrm{Qy}=24.387$. Again, the single turn phase advance was measured (without the quads and sextupoles). Figures 3 and 4 show the difference between the measured single turn phase advance and the model predication. Note the horizontal is advancing $\sim 0.03$ faster than the model and the vertical is advancing slower than the model by roughly the same amount. The circulating beam tune agrees with the single turn measurement.

\section{CONCLUSIONS}

Linear lattice properties on a permanent magnet storage ring have been measured with all tune and chromaticity correction circuits set to zero. The linear model has been further updated to include feed-down effects from the orbit offset through the magnet end-shims. The comparison between the measured and calculated tunes still show a discrepancy in tune ia $\sim 0.03$ in both planes and in chromaticity of $\mathrm{dCx} \sim+6$ units and $\mathrm{dCy} \sim+1$ units. We continue to investigate the discrepancies.

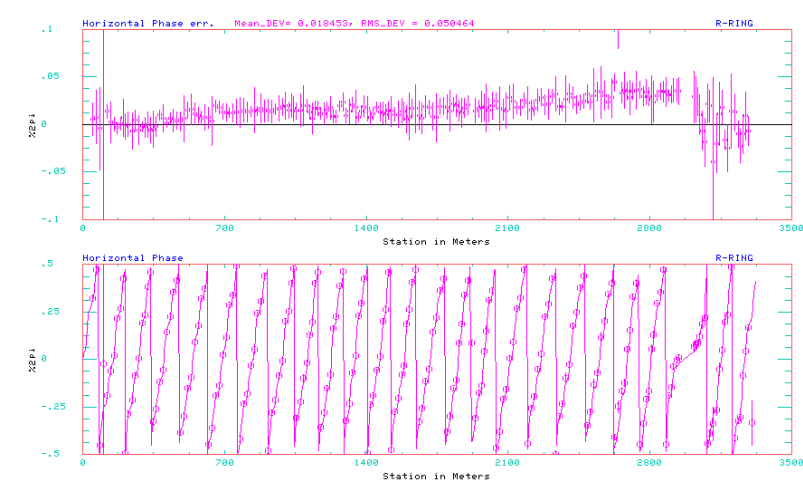

Figure 3: Horizontal phase advance after the shims were changed and heater tape was removed. Scale is $+/-0.1$

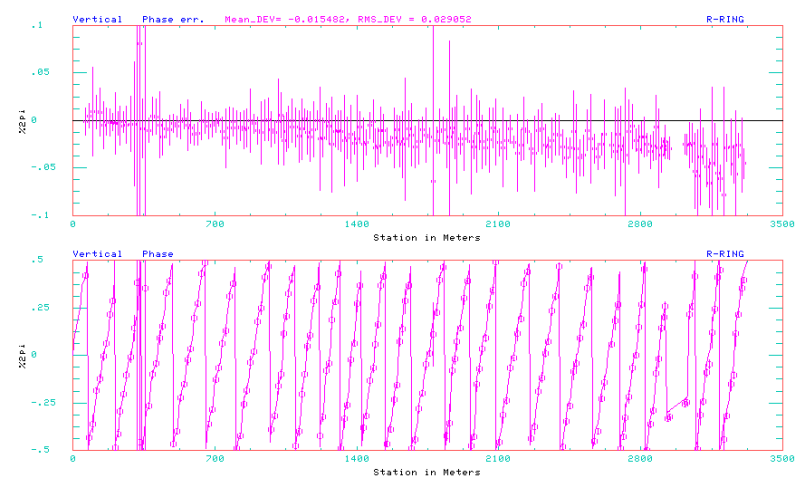

Figure 4: Vertical phase advance after the shims were changed and heater tape was removed. Scale is $+/-0.1$

\section{REFERENCES}

[1] G.P. Jackson, et.al. "Recycler Technical Design Report", FERMILAB-TM1991 (1996).

[2] D.E. Johnson, et. al., "Design and Simulation of the Antiproton Recycler Lattice", Proceedings of the 1997 Particle Accelerator Conference, p.997 (1997).

[3] B.C. Brown, et.al.,"Hybrid Permanent Magnet Gradient Dipoles for the Recycler Ring at Fermilab", FERMILAB-Conf-97/338.

[4] C.N. Brown, et.al.,"Computer Generated End Shims for Recycler Ring Magnets", Proceedings of the 1999 Particle Accelerator Conference, p.3324 (1999).

[5] S.D. Holmes, "Translating Measured Multipoles onto the Reference Orbit in Recycler Combined Function Magnet", internal publication MI NOTE-0196 (1996).

[6] B.C. Brown, et. al., "Transverse Placement of Gradient Magnets", internal publication MI NOTE-0259 (2000).

[7] M-J. Yang, Accelerator Analysis Application Program.

[8] W. Wan, et. al., "Design and Implementation of the Medium-Beta Insert of the Fermilab Recycler Ring", this conference. 